\title{
Determinação de Fatores Multiplicativos para Estimar a Produção de Leite no Dia do Controle Leiteiro, a Partir da Produção de Leite da Manhã ou da Tarde ${ }^{1}$
}

\section{Tarcísio de Moraes Gonçalves², Mário Luiz Martinez ${ }^{3}$, Cláudio Manoel Rodrigues de Melo 4 , Rui da Silva Verneque ${ }^{3}$, Antonio Ilson Gomes de Oliveira ${ }^{5}$}

RESUMO - O objetivo deste trabalho foi determinar fatores multiplicativos para estimar a produção de leite (PD) no dia do controle leiteiro, com base na produção de leite da ordenha da manhã (PM) ou da tarde (PT), obtidos de 3632 lactações de 2143 vacas da raça Gir, filhas de 336 touros, de registros entre 1986 e 1996. Ano de parto (A), duração da lactação - linear (DL), idade ao parto - linear e quadrática (I) e um fator K (possíveis interações duplas, triplas e quadrupla entre nível de manejo (baixo-alto), classe de ordem de parto (primíparas-multíparas), estação de parição (águas-seca) e nove classes de intervalo do parto ao controle leiteiro foram considerados, no modelo, para se ajustarem aos fatores multiplicativos da manhã (FM) e da tarde (FT). Foram observados efeitos significativos de A, I, DL e K sobre os fatores, à exceção do efeito da DL sobre FT, sugerindo o uso de fatores que considerem simultaneamente os efeitos contidos em K, além de A, I e DL, para se obterem estimativas mais precisas da produção de leite no dia do controle leiteiro. Os fatores multiplicativos da manhã diminuíram no decorrer da lactação, em ambas as estações, para multíparas e primíparas e animais criados em rebanhos de alto nível de manejo. No entanto, FT tendeu a aumentar, tanto para vacas como para primíparas, nas duas estações e em rebanhos que se encontram em ambos os níveis de manejo, exceto para vacas em rebanhos de baixo nível de manejo, na estação das águas, que não apresentaram comportamento constante.

Palavras-chave: bovinos de leite, fatores de ambiente, produção parcial, raça Gir

\section{Determination of Multiplicative Factors to Estimate the Milk Production at the Daily Milk Yield Control, from the Morning or Afternoon Milk Yield}

\footnotetext{
ABSTRACT - The objective of this work was to determine the multiplicative factors to estimate the milk production (DP) based on the morning (MM) or afternoon (AP) milk yield and their were obtained of 3,632 lactations from 2,143 cows of the Gir breed, daughters of 335 sires, from 1993 to 1996 records. The calving year (Y) lactation length - linear (LL), calving age - linear and quadratic (A) and a factor $\mathrm{K}$ (the possible double, triple and quadruple interactions among management level (low-high), class of parity (primiparous-multiparous), calving season (rainy-dry) and nine classes of calving to milk control interval were included in the model to fit these effects on the multiplicative morning (MF) and afternoon factors (AF). Significant effects of Y, I, LL and K on the factors, except effect of LL upon the AF were observed, suggesting the use of factors which simultaneously consider the effects contained in K, besides Y, A and LL, to obtain more precise estimates of milk yield on the day of the milk control. The multiplicative factors of the morning decrease over the lactation, in both seasons, for multiparous and primiparous, and animals raised in herds of high management level. However, AF for multiparous and primiparous, in both seasons, in both level of management, trend to increase over the lactation, except for cows in herds of low management level and in the rainy season, which did not show stable behavior.

Key Words: dairy cattle, environmental factors, partial production, Gyr breed

\section{Introdução}

A produtividade da bovinocultura leiteira, nos países menos desenvolvidos, é extremamente baixa. Contudo, o consumo total de proteína de origem animal vem aumentando a cada ano; no Brasil, em 1979, a produção média de leite era 676 kg/vaca·ano (FARIA, 1996)

e a disponibilidade de leite por habitante, $78 \mathrm{~kg} / \mathrm{ano}$ (FAO, 1979). Em 1994, a produção média de leite encontrava-se em torno de $790 \mathrm{~kg} / \mathrm{vaca}$-ano (FARIA, 1996), demonstrando, assim, que a produção média nacional não evoluiu significativamente neste período.

No Brasil, a falta de utilização de metodologias eficientes de seleção, para produção de leite, entre

1 Parte da Monografia apresentada pelo terceiro autor à Universidade Federal de Lavras (UFLA), como parte das exigências do Programa Especial de Treinamento (PET/CAPES).

2 Professor do Departamento de Zootecnia da Universidade Federal de Lavras (UFLA). DZO da UFLA, Campus da UFLA, Cx. Postal 37, CEP 37200-000, Lavras - MG, e.mail - tarcisio@ufla.br

3 Pesquisador da EMBRAPA Gado de Leite

${ }^{4}$ Aluno de Mestrado em Zootecnia na UFLA.

5 Professor Titular Aposentado do DZO da UFLA e bolsista do CNPq.
} 
outras causas, tem sido atribuída ao baixo número de animais submetidos ao controle leiteiro, o que se deve, principalmente, aos transtornos causados na fazenda pelo controle leiteiro e ao alto custo.

Entretanto, a cada dia aumenta o número de interessados em controlar a produção de seus rebanhos. Diferentes formas e métodos de controle leiteiro são preconizados e cada país adota sistema próprio. Observa-se que a tendência é diminuir a freqüência de controles, para economizar e também facilitar o trabalho das associações de controle leiteiro (LEDIC et al., 1991).

Resultados obtidos por diferentes autores, em diversos países, na estimativa da produção de leite de uma vaca, demonstraram não haver concordância em decidir qual o tipo de controle e o método de estimativa que devem ser usados para estimar a produção de leite.

Visando minimizar este o custo do controle leiteiro e aumentar o número de rebanhos controlados, propôs-se um método simplificado para estimar a produção no dia do controle leiteiro, com base em um única ordenha. Este método apresenta vantagens, quando comparado ao oficial (SCHAEFFER e RENNIE, 1976; MARTINEZ et al., 1979). Estas vantagens acarretam em aumento significativo no número de rebanhos controlados, facilitando os trabalhos de seleção para produção de leite, com a ampliação do número de animais no controle leiteiro.

Constituíram-se em objetivos deste estudo calcular fatores multiplicativos para estimar a produção de leite no dia do controle leiteiro, a partir da produção em apenas uma ordenha, e verificar os efeitos de meio que influenciam estes fatores.

\section{Material e Métodos}

Os registros utilizados no presente estudo são provenientes de 3632 lactações de 2143 vacas da raça Gir, filhas de 336 touros, com controles realizados no período de 1986 a 1996. Os animais foram distribuídos em 17 rebanhos participantes do programa de Teste de Progênie coordenado pelo Centro Nacional de Pesquisa de Gado de Leite (CNPGL), da Empresa Brasileira de Pesquisa Agropecuária (EMBRAPA), e pela Associação Brasileira dos Criadores de Gir Leiteiro (ABCGIL), com ordenha realizada duas vezes ao dia.

Foram definidas duas estações de parição - os meses de outubro a março constituíram a estação das águas (estação 1) e os de abril a setembro, a estação da seca (estação 2).

Em função da média de produção de leite em até 305 dias, para vacas que pariram com até 46 meses de idade, constituíram-se dois grupos de níveis de manejo (baixo = rebanhos com produção de leite menor que $2236 \mathrm{~kg}$ e alto = rebanhos com produção de leite maior ou igual a $2236 \mathrm{~kg}$ ). O número de observações, as médias e os respectivos erros-padrão para a produção de leite são apresentados na Tabela 1.

Foram consideradas vinte e uma classes de idade ao parto, em meses (<39, 39-41, 42-44, 45-47, 48-50, 51-53, 54-56, 57-59, 60-62, 63-65, 66-68, 69-71, 72-74, 75-80, 81-86, 87-92, 93-98,99-104, 105-110, 111-122, $123-134, \geq 135)$, nove classes de intervalo do parto ao controle leiteiro, em dias ( $\leq 30,31-60,61-90,91-120$, 121-150, 151-180, 181-210, 211-240, >240), duas classes de ordem de parto (primíparas ou multíparas), além de duas classes de nível de manejo (baixo e alto).

As alterações nas informações do arquivo para obtenção de dados consistentes e constituição das classes estudadas, assim como as análises estatísticas, foram feitas utilizando-se o programa Statistical Analysis System (SAS, 1996).

Os fatores multiplicativos considerados neste estudo foram obtidos como nos estudos de EVERETT e WADELL (1970a), a saber: FM = (PM + PT $) / \mathrm{PM} \mathrm{e}$ $\mathrm{FT}=(\mathrm{PM}+\mathrm{PT}) / \mathrm{PT}$, em que PM e PT correspondem, respectivamente, à produção de leite da ordenha da manhã e da tarde, observadas no dia do controle leiteiro.

O modelo estatístico usado para descrever os efeitos estudados que influenciam cada fator multiplicativo FM e FT foi:

\section{(MODELO 1)}

$$
\begin{aligned}
& \quad Y_{i j k l m n o p}=\mu+K_{i j k l}+A_{m}+\beta_{1}\left(D L_{i j k l m n o p}-\overline{\mathrm{D}}\right) \\
& +\beta_{2}\left(I_{i j k l m n o p}-\overline{\mathrm{I}}\right)+\beta_{3}\left(I_{i j k l m n o p}-\overline{\mathrm{I}}\right)^{2}+e_{i j k l m n o p} \\
& \text { em que }
\end{aligned}
$$

$y_{\text {ijklmnop }}=$ fator multiplicativo usado no controle leiteiro n (FM ou FT), em nível de manejo i, numa ordem de parto $\mathbf{j}$, com intervalo do parto ao controle leiteiro $\mathbf{k}$, de vacas paridas na estação $\mathbf{l}$, do ano m;

$\mu \quad=$ constante inerente a cada observação;

$K_{i j k l} \quad=$ é o efeito do nível i, classe de ordem de parto j, intervalo do parto ao controle k e estação de parição 1, em que é o efeito das possíveis interações para estas subclasses;

$A_{m} \quad=$ efeito do ano de parto $\mathbf{m}$, sendo $\mathrm{m}=$ $86,87, \ldots, 96$

$D L_{i j k l m n o p}=$ duração da lactação em dias, no controle n, no nível de manejo i, na classe de ordem de parto j, com distância do parto ao controle leiteiro 
1002 Rev. bras. zootec.

$\mathrm{k}$, de vacas paridas na estação 1 e do ano m;

$\overline{\mathrm{D}} \quad=$ média da duração da lactação;

$I_{i j k l m n o p}=$ idade ao parto, em meses, no controle $n$, no nível de manejo j, na classe de ordem de parto k, com distância do parto ao controle leiteiro l, de vacas paridas na estação $\mathrm{m}$, no ano o;

I $\quad=$ média da idade ao parto;

$\beta_{1}=$ coeficiente de regressão linear, de $\mathrm{y}_{\mathrm{ijklmnop}}$, em função da duração da lactação;

$\beta_{2}$ e $\beta_{3}=$ coeficientes de regressão linear e quadrático de $y_{\text {ijklmnop }}$, em função da idade da vaca ao parto, respectivamente; e

$e_{i j k \text { lmnop }}=$ erro aleatório residual associado a cada observação.

Para descrever os fatores multiplicativos FM e FT, dentro de cada nível de manejo (Alto e Baixo), foi adotado o seguinte modelo estatístico:

(MODELO 2)

$$
\begin{gathered}
Y_{i j k l m n o p}=\mu+R_{i}+K_{j k l}+A_{m}+\beta_{1}\left(D L_{i j k l m n o p}-\right. \\
\overline{\mathrm{D}})+\beta_{2}\left(I_{i j k l m n o p}-\overline{\mathrm{I}}\right)+\beta_{3}\left(I_{i j k l m n o p}-\overline{\mathrm{I}}\right)^{2}+e_{i j k l m n o p}
\end{gathered}
$$

em que

$y_{i j k l m}=$ fator multiplicativo usado no controle leiteiro n (FM ou FT), em um rebanho i, na ordem de parto j, com intervalo do parto ao controle leiteiro $\mathbf{k}$, de vacas paridas na estação $\mathbf{l}$, do ano $\mathbf{m}$;

$\mu \quad=$ constante inerente a cada observação;

$R_{i} \quad=$ efeito do rebanho $\mathbf{i}$;

$K_{j k l}=$ é o efeito da classe de ordem de parto $\mathbf{j}$, intervalo do parto ao controle $\mathbf{k}$ e estação de parição $\mathbf{l}$, em que $\mathbf{K}_{\mathbf{j k l}}$ é o efeito das possíveis interações para estas subclasses;

$A_{m} \quad=$ efeito do ano de parto $\mathbf{m}$, sendo $\mathbf{m}=86$, $87, \ldots, 96$;

$D L_{i j k l m n}=$ duração da lactação em dias, no controle $\mathbf{n}$, no rebanho $\mathbf{i}$, na classe de ordem de parto $\mathbf{j}$, com distância do parto ao controle leiteiro $\mathbf{k}$, de vacas paridas na estação $\mathbf{l}$ e no ano m;

$\overline{\mathrm{D}} \quad$ = média da duração da lactação;

$I_{i j k l m n}=$ idade ao parto, em meses, no controle $\mathbf{n}$, no rebanho i, na classe de ordem de parto $\mathbf{j}$, com distância do parto ao controle leiteiro $\mathbf{k}$, de vacas paridas na estação $\mathbf{l}$, no ano $\mathbf{m}$;

$\overline{\mathrm{I}} \quad=$ média da idade ao parto;

$\beta_{1}=$ coeficiente de regressão linear, de $Y_{i j k l m n}$, em função da duração da lactação;

$\beta_{2}$ e $\beta_{3}=$ coeficientes de regressão linear e quadrático de $Y_{i j k l m n}$, em função da idade da vaca ao parto, respectivamente; e

$e_{i j k l m n o p}=$ erro aleatório residual associado a cada observação.

Os fatores multiplicativos FM e FT obtidos por intermédio da solução do MODELO 2 foram suavizados pela utilização de uma equação polinomial de primeiro grau $D=\beta_{0}+\beta_{1} X$, em que

em que $\mathbf{X}$ é a média, em dias, dentro de cada classe de intervalo do parto ao controle leiteiro, consideradas neste estudo, e $\boldsymbol{\beta}_{\mathbf{0}}$ e $\boldsymbol{\beta}_{\mathbf{1}}$ são parâmetros do polinômio.

\section{Resultados e Discussão}

O resumo da análise de variância para os fatores multiplicativos, obtidos por intermédio da solução dos modelos 1 e 2, encontra-se na Tabela 2. Verifica-se que todas as causas de variação consideradas exerceram influência significativa $(\mathrm{P}<0,01)$ sobre os fatores multiplicativos calculados, exceto a duração da lactação sobre os fatores multiplicativos, para estimativa da produção de leite no dia do controle leiteiro, a partir da produção de leite da ordenha da tarde.

$O$ efeito do ano de parição sobre os fatores multiplicativos é resultante de todas as alterações que ocorrem, de ano para ano, sobre a produção de leite. Estas alterações podem ser causadas por variações climáticas que influem, diretamente, na disponibilidade e no valor nutritivo das pastagens, da alimentação e do controle sanitário e reprodutivos dos rebanhos, ou, ainda, de variações genéticas nos rebanhos, seja por seleção, cruzamentos ou por compra e venda de animais, principalmente reprodutores. Outra importante causa de variação na produção de leite no decorrer dos anos é a política governamental relacionada à produção leiteira.

Influência de ano de parição sobre a produção de leite foi observada por NOBRE(1983), ALVES (1984), POLASTRE et al. (1987), FREITAS (1988), COMERLATO (1991) e CAMARGO (1994), em

Tabela 1 - Média e desvio-padrão da produção de leite em até 305 dias, por nível de manejo

Table 1 - Mean and standard deviation for milk production at up to 305 days by management level

Nível de manejo № observação Média(EP)

Managementlevel N. obsevation Means (SE)

Alto $14302 \quad 2811(7,1)$

High

Baixo

11135

$2199(7,4)$

Low 
GONÇALVES et al.

Tabela 2 - Análise de variância dos fatores FM e MT

Table 2 - Analysis of variance of the MF and AF

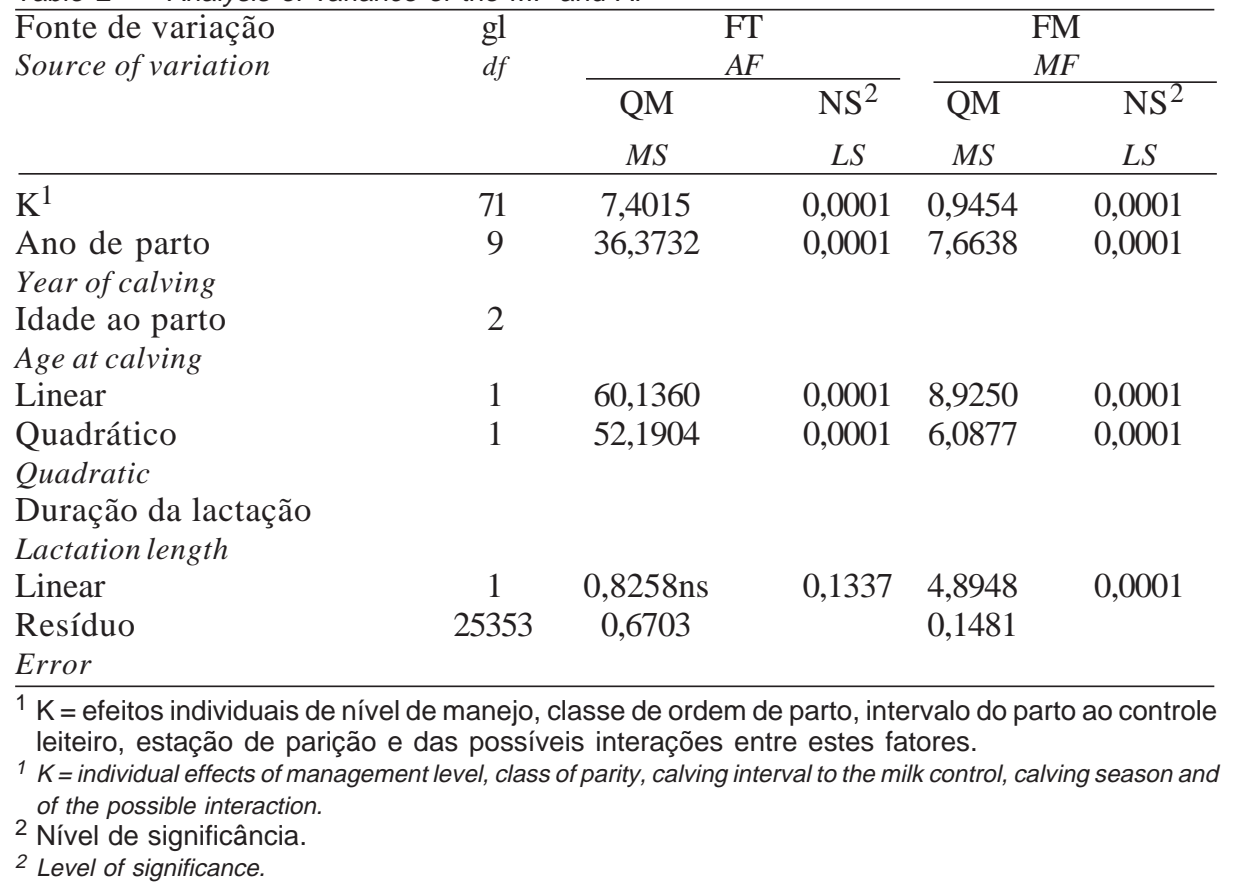

rebanhos mestiços, sendo as variações de ano para ano, nas médias de produções de leite, atribuídas a esforços de seleção para maiores produções, associados às melhoras em manejo, ambiente e níveis tecnológicos. Resultados semelhantes foram observados por ALBUQUERQUE et al. (1984), ao estudarem animais da raça Gir. PANDA e SADHU (1983) e BHUSARI et al. (1985), entretanto, não observaram influência do ano de parto sobre a produção de leite.

Pela análise da Tabela 2, pode-se inferir que houve efeito linear e quadrático $(\mathrm{P}<0,01)$ de idade da vaca ao parto sobre os fatores multiplicativos. Este efeito pode ser atribuído ao fato de vacas mais novas tenderem a produzir menos leite, o que ocorre porque animais jovens, além de estarem produzindo, estão também em crescimento, o que leva à maior exigência nutricional e menor disponibilidade de nutrientes para produção de leite. Este resultado está em concordância com os de ROJAS VIDAL (1986), JUNQUEIRA FILHO et al. (1992), MADALENA et al. (1983), POLASTRE (1985), POLASTRE et al. (1987) e MILAGRES et al. (1988), os quais observaram influência da idade ao parto sobre a produção de leite. Também, SCHAEFFER e RENNIE (1976), GONÇALVES e MARTINEZ (1995) e GONÇALVES et al. (1996) observaram efeito quadrático da idade sobre os fatores multiplicativos, para estimar a produção de leite no dia do controle leiteiro.

A duração da lactação influenciou de forma linear os fatores da manhã, porém o mesmo não se verificou no fator da tarde. Este resultado pode ser atribuído ao fato de as produções da tarde, em média, variarem menos com o decorrer da lactação. O efeito significativo da duração do período de lactação sobre os fatores multiplicativos, observado no presente estudo, é resultante da influência desta fonte de variação sobre a produção de leite.

Os efeitos individuais de nível de manejo, classe de ordem de parto, intervalo de parto ao controle e estação de parição, assim como o efeito das possíveis interações entre estes fatores $(\mathrm{K})$, influenciaram $(\mathrm{P}<0,01)$ os fatores multiplicativos estudados. Assim, sugere-se a utilização de fatores multiplicativos, para estimar a produção de leite no dia do controle leiteiro, corrigidos simultaneamente para estas variações.

Observou-se influência da duração da lactação sobre a produção de leite; resultados que concordam com os observados por REIS (1977), VERNEQUE (1982), CARDOSO et al. (1982), COELHO et al. (1983) e RAMOS (1984), estudado lactações de vacas da raça Gir, e FREITAS (1988), estudando lactações de animais mestiços, sendo as produções mais altas verificadas em longos períodos de lactação e as baixas, em períodos curtos de lactação. 
1004 Rev. bras. zootec.

Tabela 3 - Fatores multiplicativos, corrigidos simultaneamente para intervalo do parto ao controle leiteiro, nível de manejo e estação de parição, para estimar a produção diária de leite, em função da produção da manhã, em vacas

Table 3 - Multiplicative factors, simultaneously corrected for calving to milk control interval, management level and calving season to estimate the dairy production on the morning production, in cows

\begin{tabular}{lccccc}
\hline \multirow{2}{*}{$\begin{array}{l}\text { Intervalo parto- } \\
\text { controle leiteiro }\end{array}$} & \multicolumn{3}{c}{$\begin{array}{c}\text { Nível de manejo } \\
\text { Management level }\end{array}$} \\
\cline { 2 - 3 } $\begin{array}{l}\text { Calving - milk } \\
\text { control interval }\end{array}$ & \multicolumn{2}{c}{$\begin{array}{c}\text { Alto } \\
\text { High }\end{array}$} & & \multicolumn{2}{c}{$\begin{array}{c}\text { Baixo } \\
\text { Low }\end{array}$} \\
\cline { 2 - 3 } \cline { 5 - 6 } & $\begin{array}{c}\text { Seca } \\
\text { Dry season }\end{array}$ & $\begin{array}{c}\text { Águas } \\
\text { Rainy season }\end{array}$ & & $\begin{array}{c}\text { Seca } \\
\text { Dry season }\end{array}$ & $\begin{array}{c}\text { Águas } \\
\text { Rainy season }\end{array}$ \\
\hline$<30$ & 1,7793 & 1,7170 & & 1,7267 & 1,6793 \\
$31-60$ & 1,7562 & 1,7175 & & 1,7276 & 1,6962 \\
$61-90$ & 1,7343 & 1,7163 & & 1,7272 & 1,7101 \\
$91-120$ & 1,7137 & 1,7135 & & 1,7256 & 1,7204 \\
$121-150$ & 1,6948 & 1,7090 & & 1,7227 & 1,7270 \\
$151-180$ & 1,6779 & 1,7028 & & 1,7184 & 1,7297 \\
$181-210$ & 1,6626 & 1,6948 & & 1,7128 & 1,7287 \\
$211-240$ & 1,6493 & 1,6852 & & 1,7060 & 1,7238 \\
$>240$ & 1,6275 & 1,6600 & & 1,6870 & 1,7018 \\
\hline
\end{tabular}

Tabela 4 - Fatores multiplicativos corrigidos simultaneamente para intervalo do parto ao controle leiteiro, nível de manejo e estação de parição, para estimar a produção diária de leite, em função da produção da manhã, em novilhas

Table 4 - Multiplicative factors, simultaneously corrected for calving to milk control interval, management level and calving season to estimate the dairy production on the morning production, in heifers

\begin{tabular}{|c|c|c|c|c|}
\hline \multirow{3}{*}{$\begin{array}{l}\text { Intervalo parto- } \\
\text { controle leiteiro } \\
\text { Calving - milk } \\
\text { control interval }\end{array}$} & \multicolumn{4}{|c|}{$\begin{array}{l}\text { Nível de manejo } \\
\text { Management level }\end{array}$} \\
\hline & \multicolumn{2}{|c|}{$\begin{array}{l}\text { Alto } \\
\mathrm{High}\end{array}$} & \multicolumn{2}{|c|}{$\begin{array}{l}\text { Baixo } \\
\text { Low }\end{array}$} \\
\hline & $\begin{array}{c}\text { Seca } \\
\text { Dry season }\end{array}$ & $\begin{array}{c}\text { Águas } \\
\text { Rainy season }\end{array}$ & $\begin{array}{c}\text { Seca } \\
\text { Dry season }\end{array}$ & $\begin{array}{c}\text { Águas } \\
\text { Rainy season }\end{array}$ \\
\hline$\overline{<30}$ & 1,8057 & 1,7644 & 1,7350 & 1,7282 \\
\hline $31-60$ & 1,7893 & 1,7514 & 1,7382 & 1,7333 \\
\hline $61-90$ & 1,7739 & 1,7402 & 1,7402 & 1,7373 \\
\hline $91-120$ & 1,7599 & 1,7312 & 1,7409 & 1,7401 \\
\hline $121-150$ & 1,7474 & 1,7244 & 1,7402 & 1,7417 \\
\hline $151-180$ & 1,7366 & 1,7200 & 1,7382 & 1,7419 \\
\hline $181-210$ & 1,7273 & 1,7180 & 1,7348 & 1,7409 \\
\hline $211-240$ & 1,7197 & 1,7182 & 1,7301 & 1,7385 \\
\hline$>240$ & 1,7092 & 1,7261 & 1,7160 & 1,7297 \\
\hline
\end{tabular}

Nas Tabelas 3 e 4, são apresentados os fatores multiplicativos para estimar a produção diária de leite, a partir das produções da manhã, e nas Tabelas 5 e 6 , os fatores multiplicativos para estimar a produção diária de leite, a partir das produções da tarde, e os fatores multiplicativos para multíparas (3 e 5) e primíparas (4 e 6).

Estes fatores multiplicativos calculados para estimar a produção de leite no dia do controle leiteiro, em função de nível de manejo, intervalo do parto ao controle leiteiro, classe de ordem de parto e estação de parição, são desprovidos de qualquer causa de variação inclusa nos modelos estatísticos 1 e 2 . A estimativa da produção diária de leite foi obtida multiplicando-se a produção da manhã ou tarde pelo fator correspondente a nível de manejo (baixo ou alto), classe de ordem de parto (primíparas ou multíparas), estação de parição (seca ou águas) e intervalo do parto ao controle leiteiro(IPCL), em dias, no qual o animal se encontra.

Observa-se que os fatores multiplicativos para estimar a produção diária de leite no dia do controle 
GONÇALVES et al.

Tabela 5 - Fatores multiplicativos corrigidos simultaneamente para intervalo do parto ao controle leiteiro, nível de manejo e estação de parição, para estimar a produção diária de leite, em função da produção da tarde, em vacas

Table 5 - Multiplicative factors, simultaneously corrected for calving to milk control interval, management level and calving season to estimate the dairy production on the afternoon production, in cows

\begin{tabular}{|c|c|c|c|c|}
\hline \multirow{5}{*}{$\begin{array}{l}\text { Intervalo parto- } \\
\text { controle leiteiro } \\
\text { Calving-milk } \\
\text { control interval }\end{array}$} & \multicolumn{4}{|c|}{$\begin{array}{l}\text { Nível de manejo } \\
\text { Management level }\end{array}$} \\
\hline & \multirow{2}{*}{\multicolumn{2}{|c|}{$\begin{array}{l}\text { Alto } \\
\text { High }\end{array}$}} & \multirow{2}{*}{\multicolumn{2}{|c|}{$\begin{array}{l}\text { Baixo } \\
\text { Low }\end{array}$}} \\
\hline & & & & \\
\hline & Seca & Águas & Seca & Águas \\
\hline & Dry season & Rainy season & Dry season & Rainy season \\
\hline$<30$ & 2,5340 & 2,5413 & 2,6519 & 2,6582 \\
\hline $31-60$ & 2,5305 & 2,5408 & 2,6370 & 2,6402 \\
\hline $61-90$ & 2,5404 & 2,5516 & 2,6274 & 2,6294 \\
\hline $91-120$ & 2,5644 & 2,5744 & 2,6235 & 2,6267 \\
\hline $121-150$ & 2,6026 & 2,6094 & 2,6257 & 2,6321 \\
\hline $151-180$ & 2,6548 & 2,6561 & 2,6338 & 2,6458 \\
\hline $181-210$ & 2,7219 & 2,7156 & 2,6481 & 2,6680 \\
\hline $211-240$ & 2,8023 & 2,7864 & 2,6683 & 2,6982 \\
\hline$>240$ & 3,0129 & 2,9703 & 2,7289 & 2,7866 \\
\hline
\end{tabular}

Tabela 6 - Fatores multiplicativos, corrigidos simultaneamente para intervalo do parto ao controle leiteiro, nível de manejo e estação de parição, para estimar a produção diária de leite, em função da produção da tarde, em novilhas

Table 6 - Multiplicative factors, simultaneously corrected for calving to milk control interval, management level and calving season to estimate the dairy production on the afternoon production, in heifers

Intervalo parto -

controle leiteiro

Calving-milk

control interval

Nível de manejo

Management level

\begin{tabular}{lcccc} 
& Dry season & Rainy season & Dry season & Rainy season \\
\hline$<30$ & 2,4178 & 2,4089 & 2,6565 & 2,6405 \\
$31-60$ & 2,4206 & 2,4551 & 2,6371 & 2,6270 \\
$61-90$ & 2,4317 & 2,5007 & 2,6233 & 2,6188 \\
$91-120$ & 2,4516 & 2,5448 & 2,6159 & 2,6161 \\
$121-150$ & 2,4803 & 2,5870 & 2,6151 & 2,6193 \\
$151-180$ & 2,5174 & 2,6267 & 2,6209 & 2,6284 \\
$181-210$ & 2,5637 & 2,6646 & 2,6335 & 2,6434 \\
$211-240$ & 2,6182 & 2,6999 & 2,6525 & 2,6642 \\
$>240$ & 2,7580 & 2,7658 & 2,7127 & 2,7255 \\
\hline
\end{tabular}

leiteiro obtidos em função do controle da manhã, tanto para primíparas como para multíparas, nas duas estações de parição, quando os animais se encontravam em rebanhos de alto nível de manejo, diminuíram no decorrer da lactação, enquanto os fatores para animais que se encontravam em rebanhos de baixo nível de manejo, em ambas as estações de parição, não apresentaram comportamento constante. No entanto, os fatores da tarde, tanto para vacas como para novilhas, nas duas estações de parição e em rebanhos que se encontravam em ambos os níveis de manejo, tenderam a aumentar no decorrer da lactação. O comportamento diferenciado em cada uma das situações estudadas foi atribuído ao comportamento da produção de leite diária, no decorrer da lactação.

\section{Conclusões}

Fatores multiplicativos, corrigidos simultaneamente para intervalo do parto ao controle leiteiro, nível de manejo, estação de parição e classe de ordem de parto, a fim de estimar a produção de leite, podem ser usados para estimar a produção de leite no dia do controle leiteiro, a partir a produção de leite de apenas uma ordenha.

Ano de parto, idade da vaca ao parto, duração da 
lactação e efeitos individuais de nível de manejo, classe de ordem de parto, intervalo do parto ao controle leiteiro, estação de parição, assim como efeito das possíveis interações entre estes fatores, influíram nas estimativas dos fatores, à exceção para efeito da duração da lactação sobre os fatores multiplicativos da tarde.

\section{Referências Bibliográficas}

ALBUQUERQUE, L.G., LOBO, R.B., GANDINE, C.H. et al. Produções parciais acumuladas de leite em rebanho Gir. Influência de efeito não-genético. In: REUNIÃO ANUAL DA SOCIEDADE BRASILEIRA DE ZOOTECNIA, 21, 1984, Belo Horizonte. Anais... Belo Horizonte: SBZ, 1984. p.47.

ALVES, A.J.R. Influência de fatores genéticos e de meio sobre a produção de leite de vacas mestiças. Viçosa: UFV, 1984. 90p. Dissertação (Mestrado em Melhoramento Genético) Universidade Federal de Viçosa, 1984.

BHUSARI, M.B., TAJANE, K.R., DEB, R.N. 1985.The probable components of variation in milk yield of Sahiwal. Ind. Vet. J., 62(2):151-154.

CAMARGO, A.J.R. Estudo de algumas características produtivas e reprodutivas de um rebanho mestiço HolandêsZebu no Estado do Rio de Janeiro: Viçosa, MG, UFV, 1994. 78p. Dissertação (Mestrado em Zootecnia) - Universidade Federal de Viçosa, 1994.

CARDOSO, V.L., PIRES, F.L., FREITAS, M.A.R. et al. Aspectos da produção de um rebanho da raça Gir em seleção leiteira. In: REUNIÃO ANUAL DA SOCIEDADE BRASILEIRA DEZOOTECNIA, 19, 1982, Piracicaba. Anais .... Piracicaba: SBZ, 1982. p.2-3-204.

COELHO, M.J.A., PRIMO, G.B., LEITE, P.R.M. et al. Fatores ambientais e genéticos como causa de variação no período de lactação da raça Gir. I. Produção de leite In: REUNIÃO ANUAL DA SOCIEDADE BRASILEIRA DE ZOOTECNIA, 20, 1983, Pelotas. Anais .... Pelotas: SBZ, 1983. p.223.

COMERLATO, L.R. Estudo de fatores genéticos e de meio em características produtivas e reprodutivas de um rebanho mestiço Europeu: Zebu. Viçosa: UFV, 1991. 116p. Dissertação (Mestrado em Melhoramento Genético) Universidade Federal de Viçosa, 1991.

EVERETT, R.W., WADELL, L.H. 1970. Sources of variation affecting the difference between morning and evening dairy milk production. J. Anim. Sci., 53(10):1424-1429.

FARIA, V.P. 1996. O que está realmente acontecendo na pecuária de leite do país? (Parte I). Boletim do Leite, 3(30):1-4.

FOOD AND AGRICULTURE ORGANIZATION OF THE UNITED NATIONS. 1979. Production Year Book. Rome, FAO, v.33. 309 p.

FREITAS, A.F. Fatores genéticos e de meio em algumas características produtivas de um rebanho leiteiro mestiço. Viçosa: UFV, 1988. 117p. Tese (Doutorado em Zootecnia) - Universidade Federal de Viçosa, 1988.

GONÇALVES, T.M., MARTINEZ, M.L. Utilização das produções de leite das vacas da raça Nelore. In: REUNIÃO ANUAL DA SOCIEDADE BRASILEIRA DE ZOOTECNIA, 33, 1995, Brasília. Anais... Brasília: SBZ 1995, p.727-728.

GONÇALVES, T.M., MELO, C.M.R., MARTINEZ, M.L. et al. Fatoresmultiplicativos para estimar a produção de leite no dia do controle, em vacas da Raça Nelore. In: REUNIÃO ANUAL DA SOCIEDADE BRASILEIRA DE
ZOOTECNIA, 33, 1996, Fortaleza. Anais... Fortaleza: SBZ 1996, p.48-50.

JUNQUEIRA FILHO, G.N.J., VERNEQUE, R.S., LEMOS, A.M. et al. 1992. Fatores Fisiológicos e de meio sobre a produção de leite por vacas mestiças leiteiras no CNPGL/ EMBRAPA. Pesq. Agropec. Bras., 27(1):153-162.

LEDIC, I.L., SOBRINHO, E.B., TUPY, O. 1991. Comparação de alguns métodos de previsão de leite com base em controles semanais, quinzenais, mensais e bimensais. Pesq. Agropec. Bras., 26(8):1127-1137.

MADALENA, F.E., VALENTE, J., TEODORO, R.L. et al. 1983. Produção de leite e intervalo entre partos de vacas HPB e mestiças HPB-Gir num alto nível de manejo. Pesq. Agropec. Bras., 18(2):195-200.

MARTINEZ, M.L., FREITAS, A.F., MADALENA, F.E. 1979. Comparação de métodos para estimar a produção de leite com base em controle mensais. Pesq. Agropec. Bras., 14(3):243-250.

MILAGRES, J. C., ALVES, A. J. R., PEREIRA, J. C. et al. 1988. Influência de fatores genéticos e de meio sobre a produção de leite de vacas mestiças das raças Holandesa, Schwyz e Jersey com Zebu. II. Produção de leite. R. Soc. Bras. Zootec., 17(4):341-357.

NOBRE, P. R. C. Fatores genéticos e de meio em características produtivas e reprodutivas do rebanho leiteiro da UFV, Estado de Minas Gerais. Viçosa: UFV, 1983. Dissertação (Mestrado em Zootecnia) - Universidade Federal de Viçosa, 1983.

PANDA, P.B., SADHU, D.P. 1983. Some genetic and nongenetic factors affecting milk production in cross-breeds of Holstein and Jersey with Hariana and Deshi Bengal cows. Ind Vet. J., 60(2):99-106.

POLASTRE, R. Fatores genéticos e de ambiente do desempenho de vacas mestiças Holandês-Zebu. Viçosa: UFV, 1985. 128p. Tese (Doutorado em Zootecnia) - Universidade Federal de Viçosa, 1985.

POLASTRE, R., MILAGRES, J.C., TEIXEIRA, N.M. et al. 1987. Fatores genéticos e de ambiente do desempenho de vacas mestiças Holandês-Zebu. I. Idade ao primeiro parto. $R$. Soc. Bras. Zootec., 16(3):228-232.

RAMOS, A.A. Estudo das características reprodutivas e produtivas de zebuínos leiteiros da raça Gir nos trópicos. Botucatu: UNESP, São Paulo, 1984. 224p. Tese (Livre Docência de Bovinocultura de Leite) - Universidade Estadual do Estado de São Paulo, 1984.

REIS, S.R. Fatores de variação do período de lactação e da produção de leite num rebanho mestiço. Europeu-Zebu. Belo Horizonte: UFMG, 1977. 86p. Dissertação (Mestrado em Produção Animal) - Universidade Federal de Minas Gerais, 1977.

ROJAS VIDAL, F. Influência de fatores genéticos e de meio em características produtivas e reprodutivas em dois rebanhos leiteiros no Departamento de Santa Cruz de La Sierra Bolivia. Viçosa: UFV, 1986. 105p. Dissertação (Mestrado em Zootecnia) - Universidade Federal de Viçosa, 1986.

SCHAEFFER, L. R., RENNIE, J. C. 1976. AM-PM Testing for estimating lactation yields. Can. J. Anim. Sci., 56(1):9-15.

STATISTICAL ANALYSIS SYSTEM - SAS. 1996. User's Guide: Statistics, Cary: SAS INSTITUTE. 956p.

VERNEQUE, R. S. Fatores genéticos e de meio em características produtivas e reprodutivas de um rebanho Gir leiteiro. Viçosa: UFV, Imprensa Universitária, 1982.93p. Dissertação (Mestrado em Zootecnia) - Universidade Federal de Viçosa, 1982.

Recebido em: 18/05/98

Aceito em: 30/03/99 